\title{
Acquired cystic disease of the kidneys: a hazard of long-term intermittent maintenance haemodialysis
}

\author{
M. S. DUNNILL, P. R. MILLARD, AND D. OLIVER \\ From Gibson Laboratories, Radcliffe Infirmary and Haemodialysis Unit, Oxford, UK
}

SUMMARY In the period 1968-76, necropsies were carried out on 30 patients who had been treated by long-term intermittent maintenance haemodialysis. Fourteen of these patients developed bilaterafo cystic disease of the kidney. Clinical, pathological, and radiological investigation of these patientso when they first presented did not reveal any evidence of renal cystic change. The main complications ${ }^{\circ}$ of this condition are haemorrhage and tumour formation. Six patients developed renal tumours,,$\infty$ and in five cases these were multiple. The histological appearance of these neoplasms gave noo indication as to whether they would behave in an aggressive manner. One patient died of metastatic carcinomatosis from a renal primary. The condition of acquired cystic disease of the kidney shouldes be suspected if patients on maintenance haemodialysis suffer from recurrent haematuria or are found to have enlarging kidneys.

End stage renal disease, and in particular chronic glomerulonephritis, is associated with small scarred contracted kidneys (Heptinstall, 1968; 1974). The advent of long-term intermittent haemodialysis has resulted in patients with this previously lethal condition living for years and in many instances receiving a renal allograft enabling them to return to a normal working life. An opportunity to examine the kidneys of such patients is provided not only at necropsy but also when bilateral nephrectomy is performed in order to control hypertension. We report here cystic transformation in the kidneys of these patients and point out the complications of this change.

\section{Clinical features}

During the period 1968-76, 236 patients were accepted for long-term intermittent haemodialysis. Fifty-one died and necropsies were performed on 30 . Congenital adult polycystic disease of the kidneys was excluded from this study. Fourteen patients were found at necropsy to have extensive bilateral cystic disease of the kidneys. The brief clinical details of these patients are given in the Table. The mean age at death was 47.8 years and the mean length of time on maintenance haemodialysis was 3.4 years. Although renal biopsy had been performed in only

Received for publication 10 March 1977 eight cases during the course of the original disease, there was no clinical or pathological evidence trito any of these 14 patients suffered from congenim adult polycystic disease. Radiological examination, including either intravenous or retrograde pyelography, performed when they first presented did not๊ reveal any evidence of cystic change.

In three instances there was clinical evidence of $\overrightarrow{\vec{\rho}}$ enlargement of the kidneys occurring during the period on haemodialysis. In case 3 there was pain in the loin and systemic evidence of haemorrhage as well as haematuria. At necropsy a massive left-sideç renal haematoma was found. Haematuria togethero with radiological evidence of an enlarging left kidney?. in case 7 necessitated a left-sided nephrectomy af which haemorrhage into cysts in the kidney was found in addition to a large adenocarcinoma? Bilateral nephrectomy was performed in case $11_{\supset}$ because of massive perirenal haemorrhage ando haematuria accompanied by radiological evidence of enlarging kidneys (Figs 1 and 2).

\section{Pathological findings}

GROSS APPEARANCES

The kidneys in these cases present a very variable appearance but in general both kidneys are equallye⿱ affected. Increase in size is not a necessary conse ${ }_{-}$ quence of total or almost total replacement by cystș (Fig. 3). The cysts vary in number and type. In somę 
kidneys there are a relatively few circumscribed structures, measuring up to $2 \mathrm{~cm}$ in diameter, often clearly visible on the subcapsular surface and with well defined and quite plentiful intervening parenchyma (Fig. 4), whereas in others there are numerous cysts replacing the entire renal substance. Occasionally, cysts are most striking in the medulla, often adjacent to the pelvis, or at the corticomedullary junction and are reminiscent of the appearances described in uraemic medullary cystic disease. In those cases where enlargement of the kidneys is an obvious feature (Fig. 2) tumours and haemorrhage are often but not invariably present. Tumours were found in six patients and in five instances were multiple. They are most often spherical in shape and apparently encapsulated with a greyish or yellowish cut surface. Haemorrhage may be present in the centre of the tumour. In some instances the tumours assume a papillary form, projecting into cystic spaces. Many cysts are multilocular and tumours occupying the cavity of such cysts may appear multiple when in fact they are but an extension of a single tumour into the various locules of a multilocular cyst. However, true multiple tumours are a frequent finding and can be seen in widely separated areas of the kidney.

Haemorrhage is a prominent feature of this condition and may take the form of bleeding into a cyst or tumour which can give rise to haematuria if the resultant haematoma ruptures into the renal pelvis. Alternatively, as in case 11, the haematoma may rupture into surrounding tissues and give rise to massive retroperitoneal haemorrhage.

\section{HISTOLOGY}

The renal parenchyma, as would be expected, exhibits complete disorganisation of its architecture. Glomeruli are scanty and seldom possess patent capillaries. Occasionally evidence of the original disease can be made out, and this is particularly true of membranoproliferative glomerulonephritis (Figs 5 and 6) where, although the glomerular tuft is hyalinised, lobularity is maintained. Tubules appear totally atrophic with lack of distinction between proximal and distal tubules. Interstitial fibrosis is pronounced and often remarkable for the number of iron-laden macrophages (Fig. 7). These are seen in tissue adjacent to cysts both with and without tumours and represent areas of old haemorrhage. Focal areas of calcification are present in the interstitial tissue and occasionally in the tubules. A consistent finding in all kidneys is severe arterial disease affecting vessels of all sizes with marked intimal fibrosis causing very severe luminal narrowing or total occlusion.

Histology of the cysts themselves is not particularly informative. They are lined by flattened cuboidal epithelium (Fig. 8) and do not differ in appearance whether they are in cortex or medulla. Oxalate crystals are prominent in some cyst cavities.

The tumours are of variable appearance but can be broadly classified into papillary, tubular or solid neoplasms. The papillary tumours (Fig. 9) consist of one or more layers of rather uniform cells, with small hyperchromatic nuclei, covering a thin branching vascular fibrous connective tissue stroma. Mitotic figures are rare. These tumours invariably arise from the lining epithelium of the cysts and project into the cystic cavity. Tumours exhibiting tubular differentiation fall into two categories. The first is composed of well defined tubular structures lined by uniform cells with rather basophilic cytoplasm (Fig. 10). In the second type the tubular differentiation may appear less marked and the tumour is composed of uniform cells with clear or foamy cytoplasm containing lipid (Fig. 11).

The solid type of tumour nearly always consists of clear cells though occasionally it may be made up of

Table Patients on long-term intermittent haemodialysis with cystic kidneys at necropsy

\begin{tabular}{|c|c|c|c|c|c|c|}
\hline Case & $\operatorname{Sex}$ & Age & $\begin{array}{l}\text { Time on } \\
\text { dialysis }(y r)\end{array}$ & Clinical diagnosis & $\begin{array}{l}\text { Kidney } \\
\text { weight }(g)\end{array}$ & Tumour(s) \\
\hline 1 & $\mathbf{M}$ & 41 & $0 \cdot 75$ & Chronic glomerulonephritis & 138 & None \\
\hline 2 & $\mathbf{M}$ & 53 & $1 \cdot 50$ & Essential hypertension & 182 & Multiple \\
\hline 3 & $\mathbf{M}$ & 45 & $3 \cdot 25$ & Membranoproliferative glomerulonephritis & $794^{*}$ & None \\
\hline 4 & $\mathbf{F}$ & 41 & 0.33 & Amyloidosis & 178 & None \\
\hline 5 & $\mathbf{M}$ & 47 & $1 \cdot 66$ & Chronic glomerulonephritis & 116 & None \\
\hline 6 & $\mathbf{F}$ & 47 & $3 \cdot 00$ & Renal artery stenosis & 80 & None \\
\hline 7 & $\mathbf{M}$ & 56 & $2 \cdot 00$ & Chronic pyelonephritis & $380 * *$ & Single \\
\hline 8 & $\mathbf{M}$ & 34 & $4 \cdot 00$ & Schölein-Henoch purpura & 60 & None \\
\hline 9 & $\mathbf{M}$ & 57 & $1 \cdot 00$ & Essential hypertension & 114 & None \\
\hline 10 & $\mathbf{F}$ & 58 & 6.00 & Chronic pyelonephritis & 110 & None \\
\hline 11 & $\mathbf{M}$ & 38 & $7 \cdot 00$ & Membranoproliferative glomerulonephritis & 680 & Multiple \\
\hline 12 & $\mathbf{M}$ & 41 & $7 \cdot 00$ & Chronic glomerulonephritis & 390 & Multiple \\
\hline 13 & $\mathbf{M}$ & 53 & $3 \cdot 00$ & Chronic glomerulonephritis & 156 & Multiple \\
\hline 14 & $\mathbf{M}$ & 58 & $7 \cdot 00$ & Chronic glomerulonephritis & 278 & Single \\
\hline
\end{tabular}

*Includes haematoma.

**Weight of one kidney only. Previous contralateral nephrectomy. 


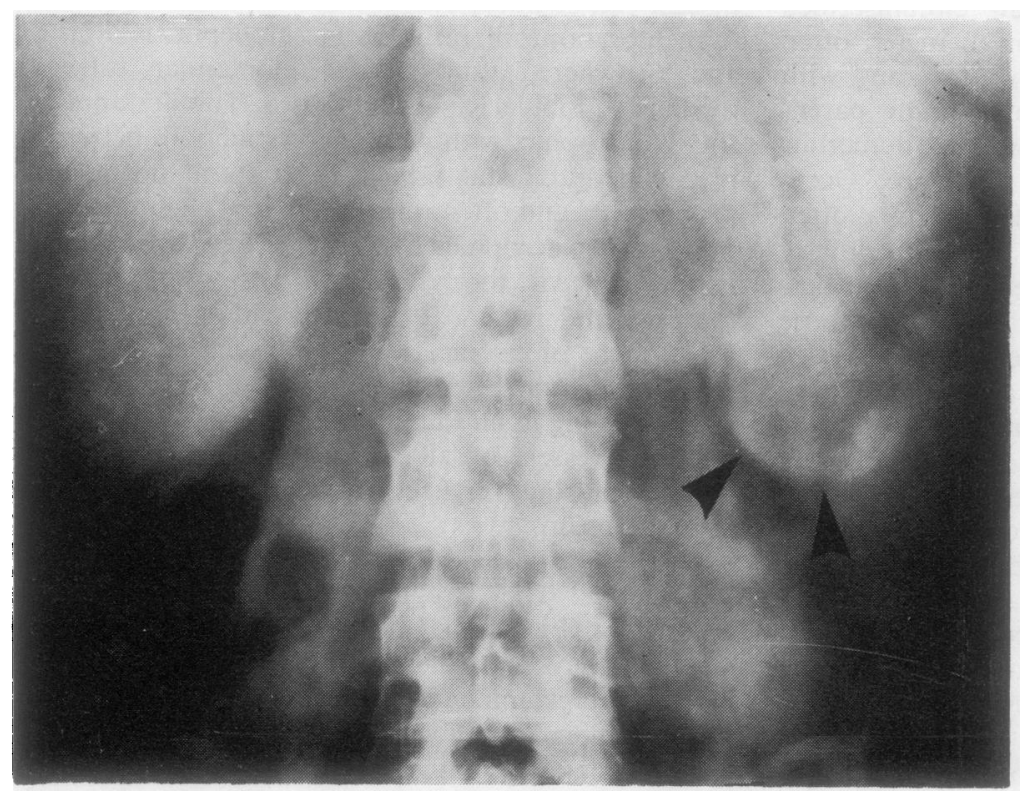

Fig. 1 Case 11. Radiograph of abdomen. Irregular outline of left renal shadow suggests cyst formation (arrowed). Focal areas of calcification can also be seen in both right and left kidneys.

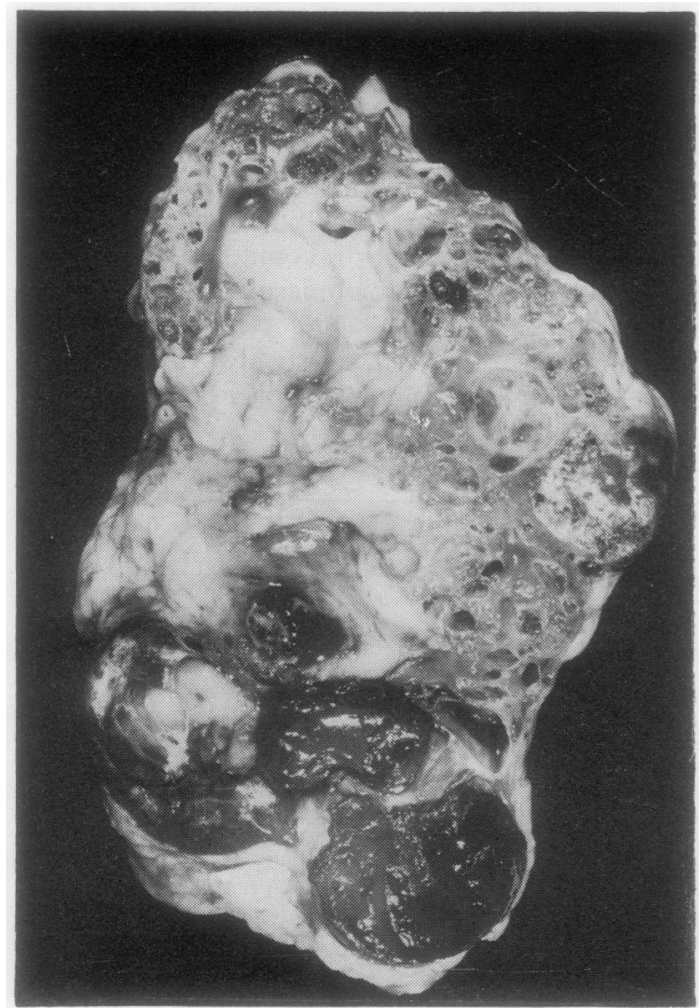

Fig. 2 Case 11. The removed left kidney. Note the haemorrhages at the lower pole, some of which are in cysts but one of which is into a tumour. A separate tumour can be seen in the mid-zone just below the capsule. The general outline of the kidney corresponds to the left kidney illustrated in the radiograph in Fig. 1 . 


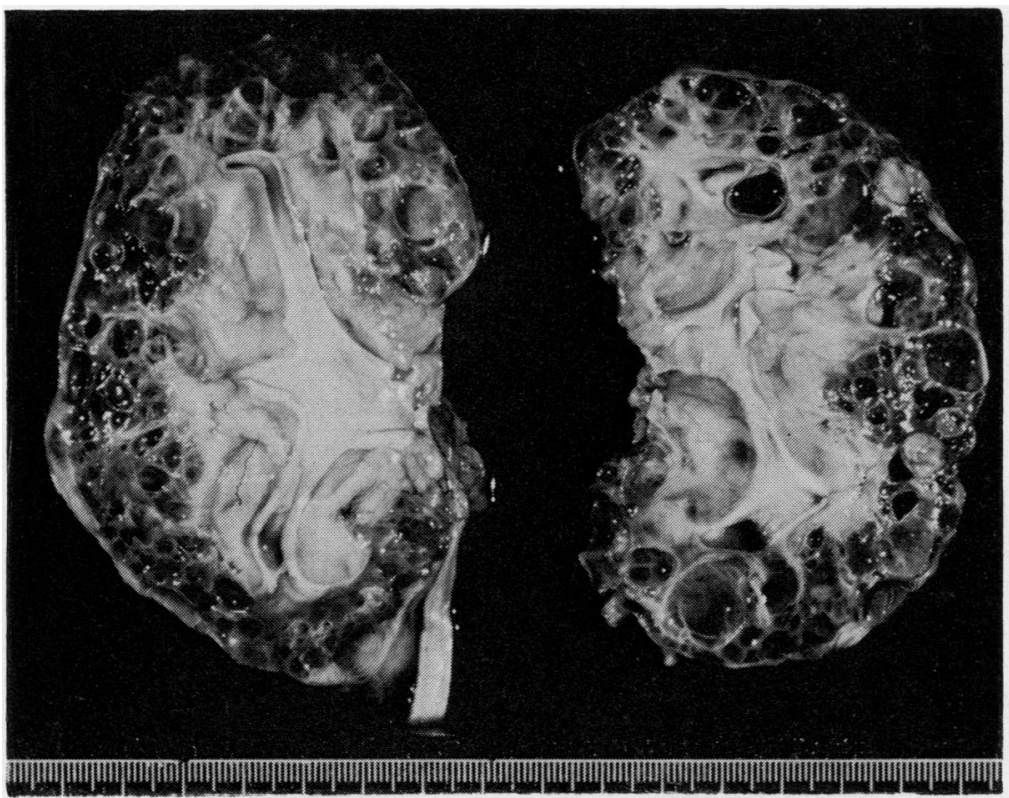

Fig. 3 Case 14. Kidneys-scale in $\mathrm{mm}$. There is total replacement by cysts but the kidneys are reduced in size.

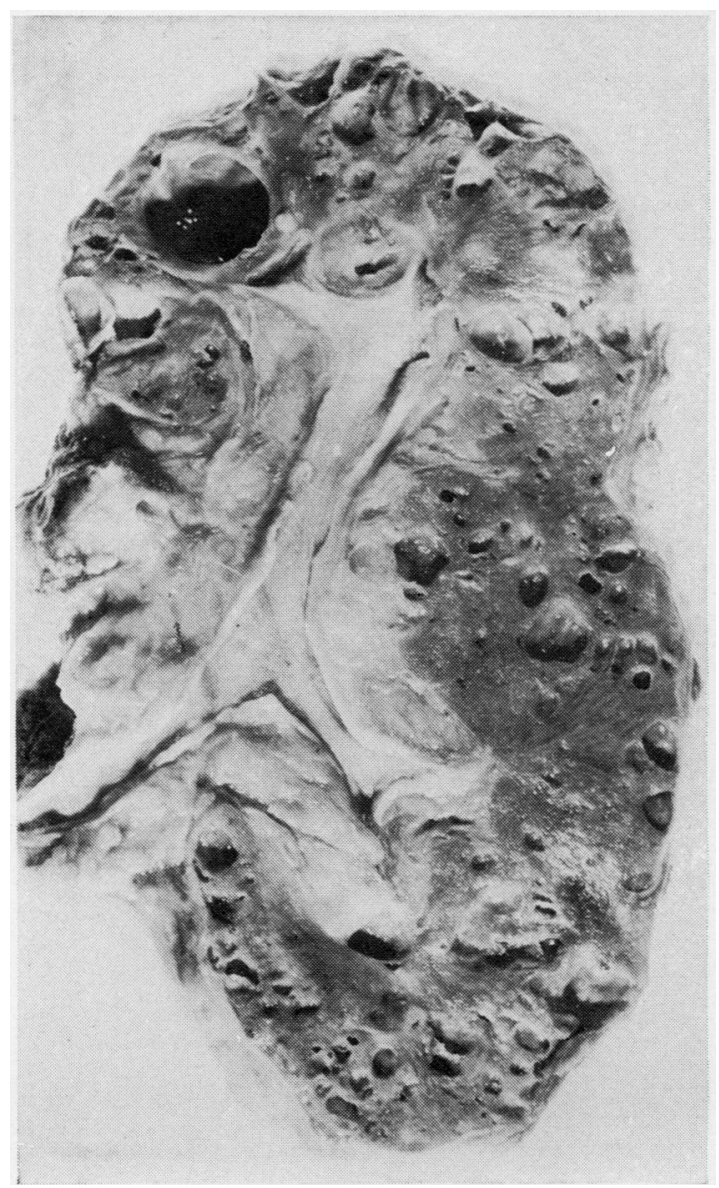

Fig. 4 Case 7. Extensive cyst formation in normal size kidneys with fairly plentiful remaining renal parenchyma. 


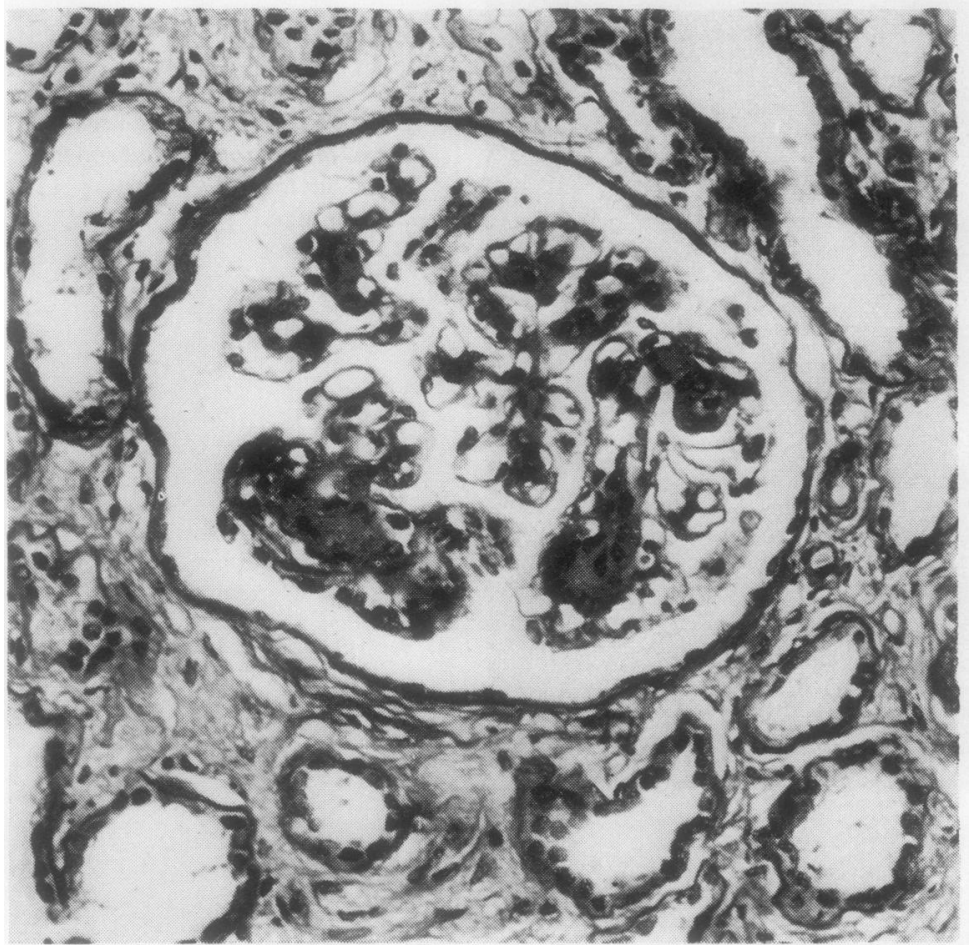

Fig. 5 Case 3. Renal biopsy taken three years before death showing a glomerulus exhibiting changes compatible with the diagnosis of membranoproliferative glomerulonephritis. Periodic-acid Schiff preparation $\times 340$.

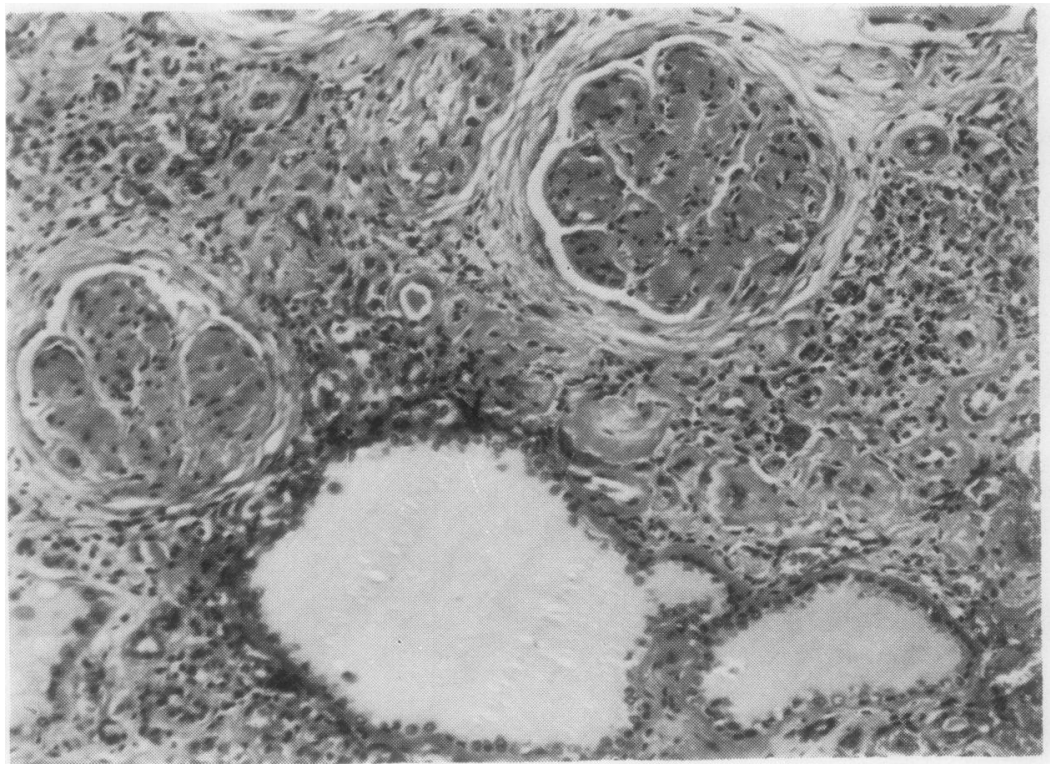

Fig. 6 Case 3. Histologica section taken from necropsy kidney showing several glomeruli with a lobulated appearance indicative of advanced membranoproliferative change. Haematoxylin and eosin $\times 170$. 


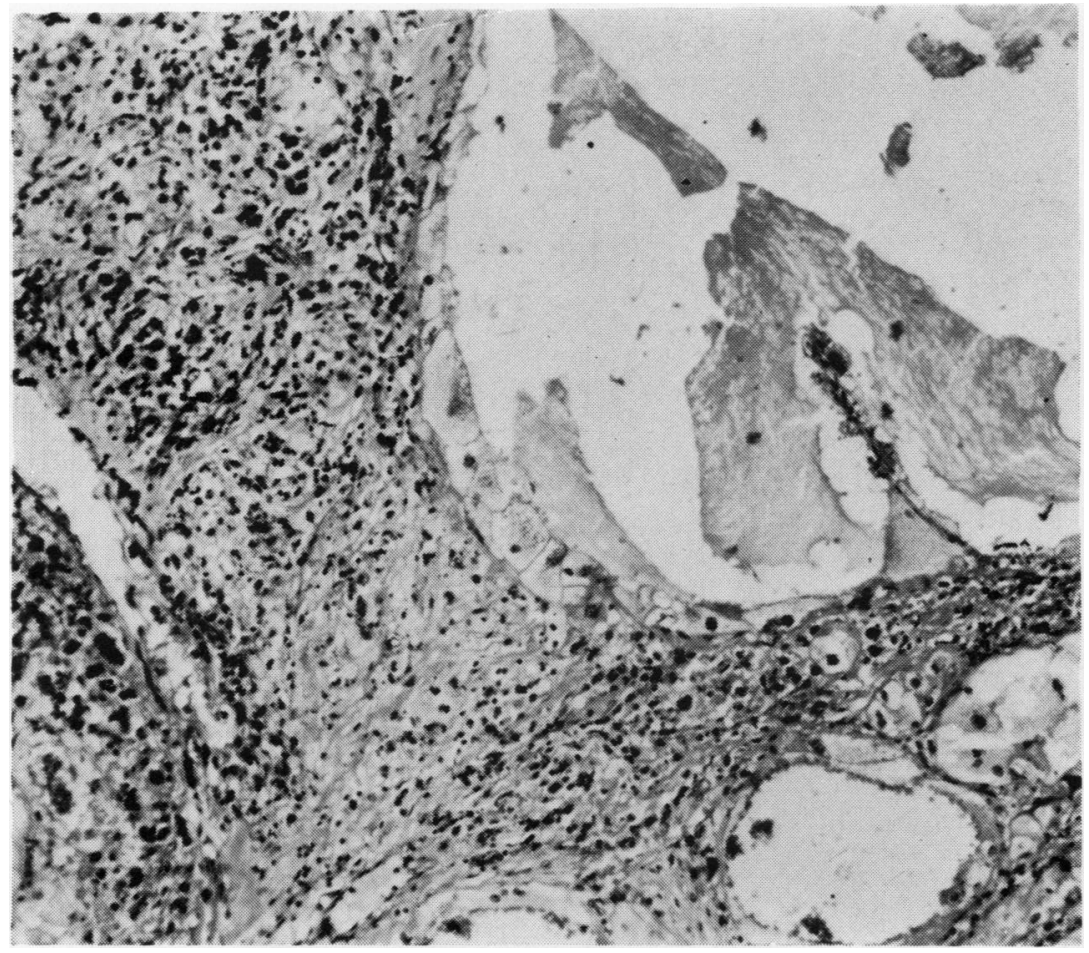

Fig. 7 Numerous ironladen macrophages present in connective tissue surrounding cysts. The cysts are lined by 'clear' cells. $H$ and $E \times$ 135.

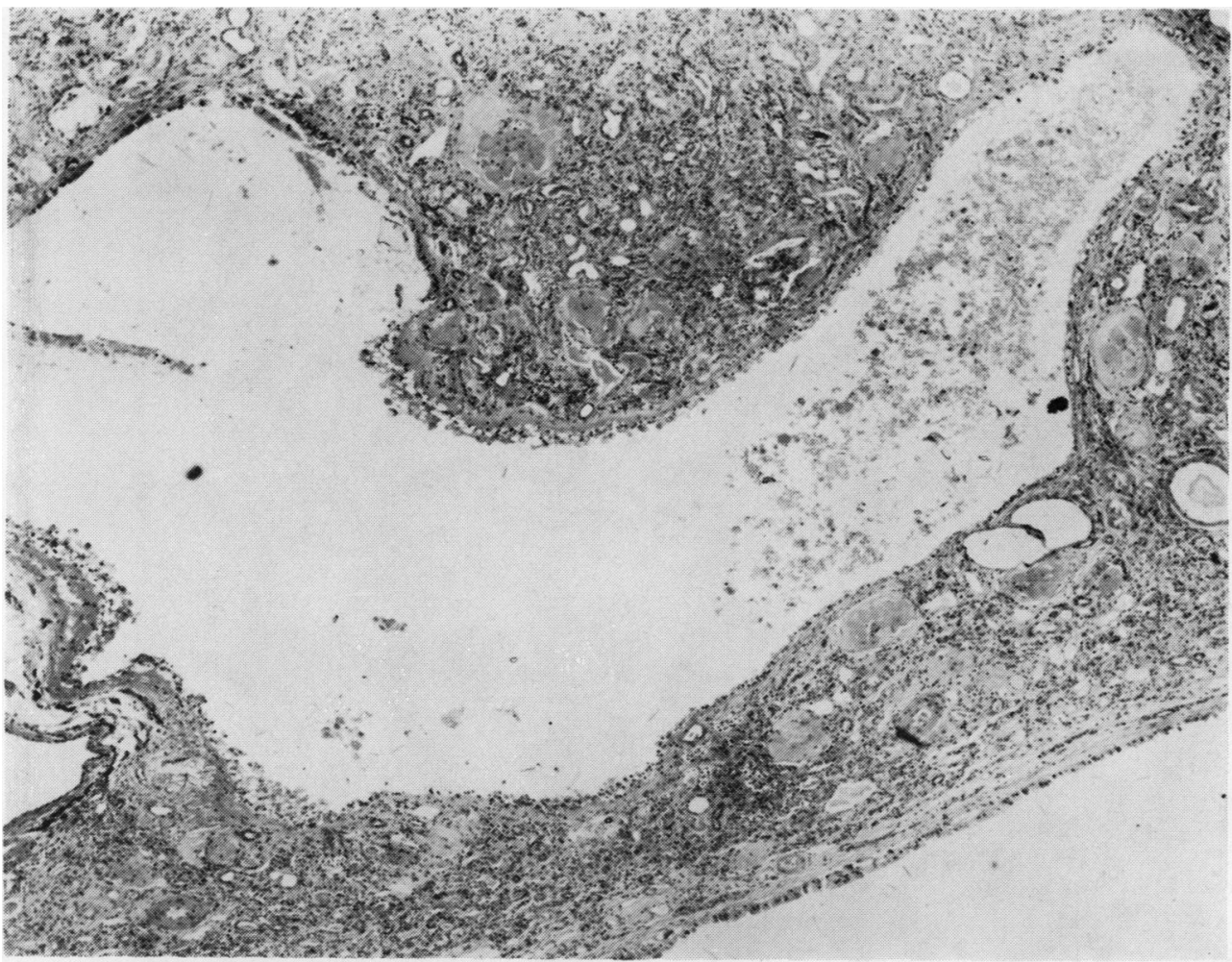

Fig. 8 Portions of the wall of two cysts showing disorganised renal parenchyma between the cyst cavities and the flattened cuboidal epithelium lining the cysts. The material in the upper cyst cavity is a mass of oxalate crystals. $H$ and $E \times 50$. 


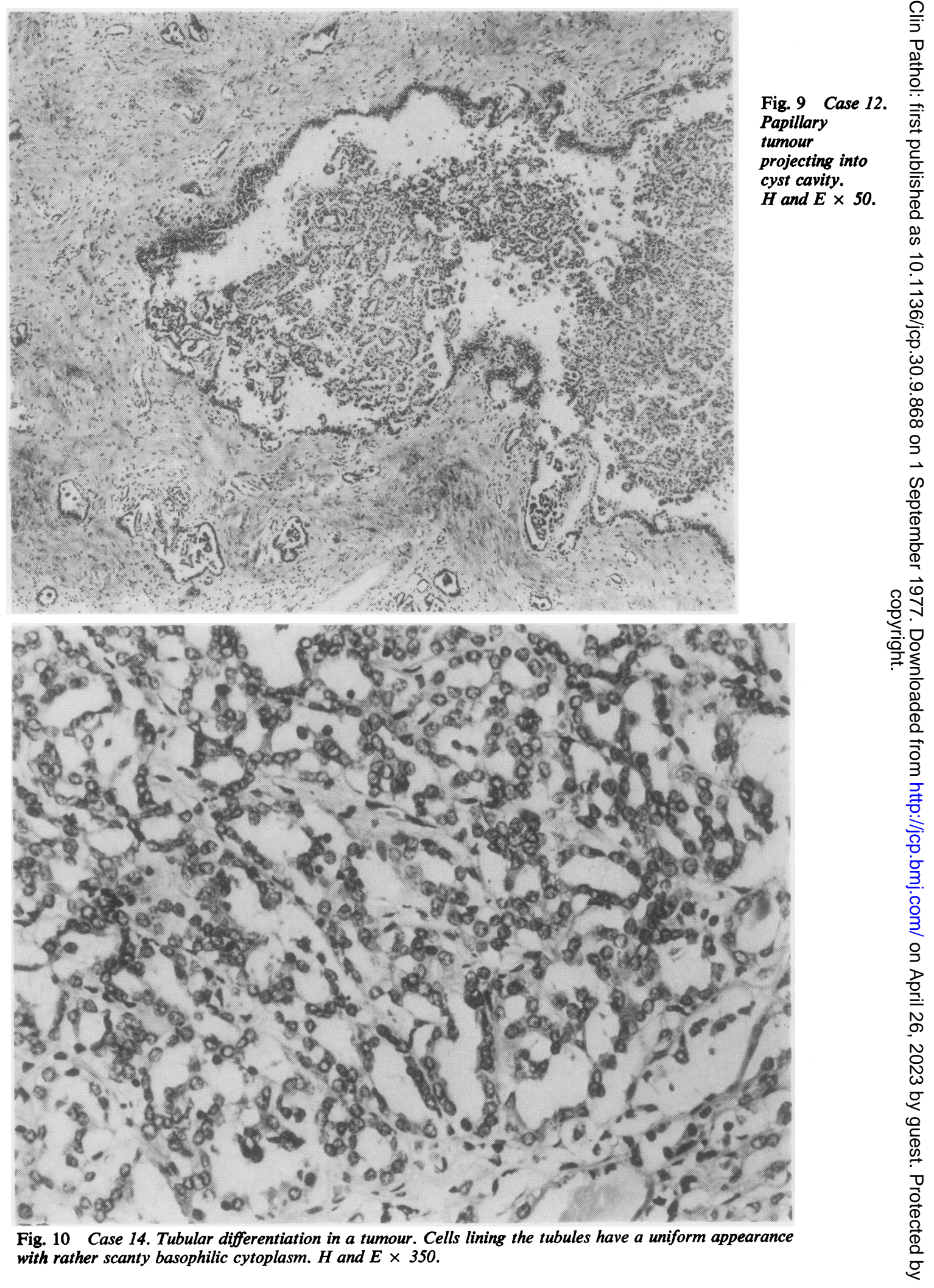




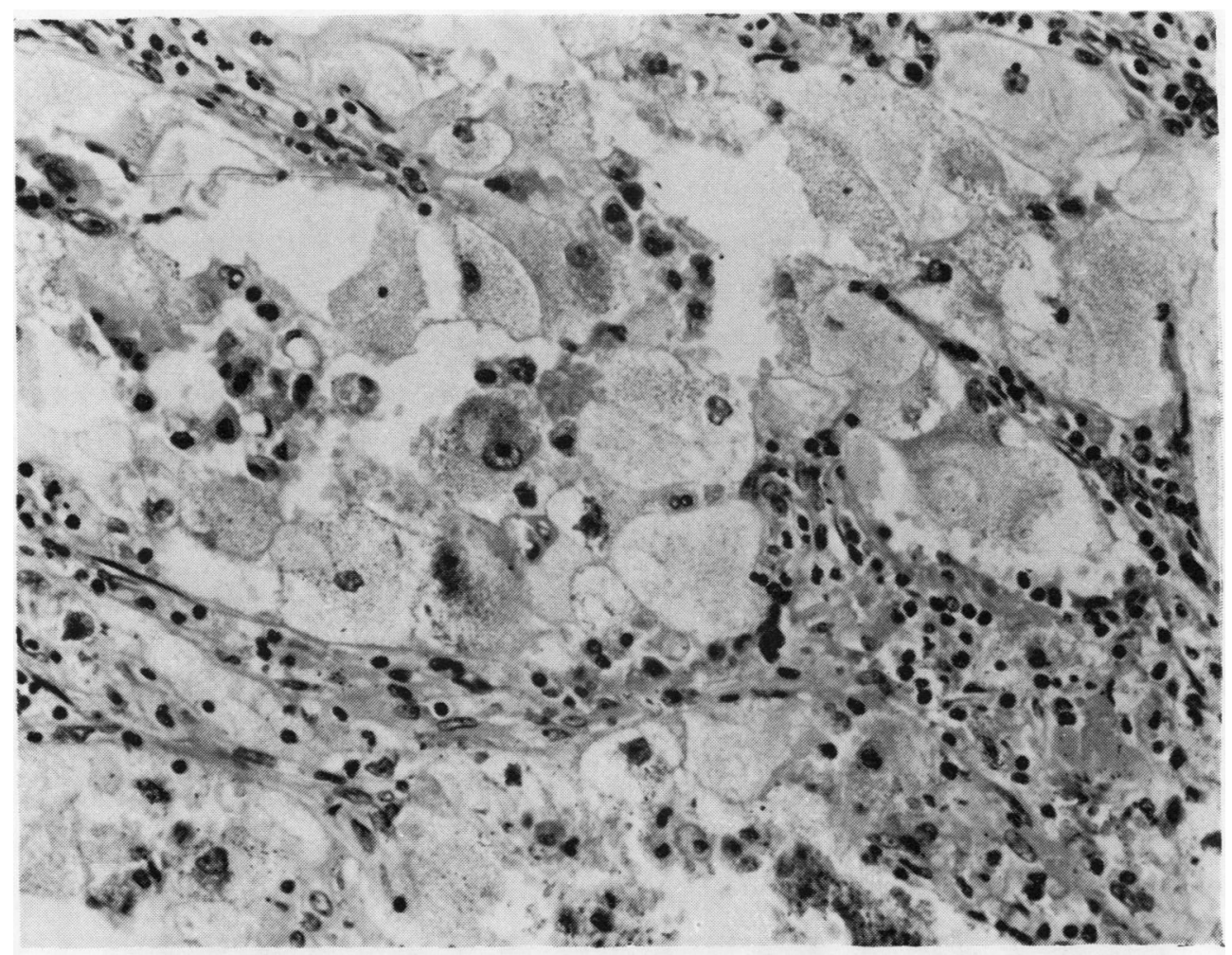

Fig. 11 Case 3. 'Foam' or 'clear' cells lining rather poorly defined tubules. $H$ and $E \times 340$.

cells with uniform, non-foamy, acidophilic cytoplasm (Fig. 12a). Tumours of more than one type may be present in any given kidney (Fig. 12b). Central areas of haemorrhage into these tumours are common.

It is extremely difficult to assess the malignant potential of any individual tumour. One of the most striking features of all these tumours is that the cytology of any single lesion is often uniform with little in the microscopic appearances to suggest malignancy, a feature well illustrated by case 7 where the patient died with disseminated carcinomatosis, including metastases in bone, from a primary clear cell adenocarcinoma.

\section{Discussion}

The advent of long-term intermittent haemodialysis and of renal transplantation has resulted in marked prolongation of life in patients with uraemia due to chronic renal disease. Unless a biopsy has been performed during the acute stages of the initial illness it may be impossible to ascertain the original pathology from examination of the end stage kidney. The observation that the end stage kidney of chronic glomerulonephritis is a small scarred contracted organ is based on findings at necropsy on patients: who had died in uraemia before dialysis treatment: became commonplace. The kidney has a limited' number of responses to abnormal stimuli and by the: time terminal uraemia is reached the scarred organ may have much the same appearance no matter what the original disease process. It may be difficult to determine even whether glomerulonephritis or pyelonephritis was the initial insult, let alone which form of glomerulonephritis was involved. Only in disorders such as amyloid, where there is a specific ultrastructural appearance, is it possible to state with conviction the underlying pathological process. It is probable that the majority of kidneys in patients on long-term haemodialysis conform to the classical contracted scarred type but in a significant number of cases the kidneys appear to undergo cystic transformation of the kind illustrated here. The pathogenesis of this change is not understood. In many instances it occurs in the absence of glomerular filtration as often these patients are anuric. It is possible that the cysts represent dilated tubules or col- 


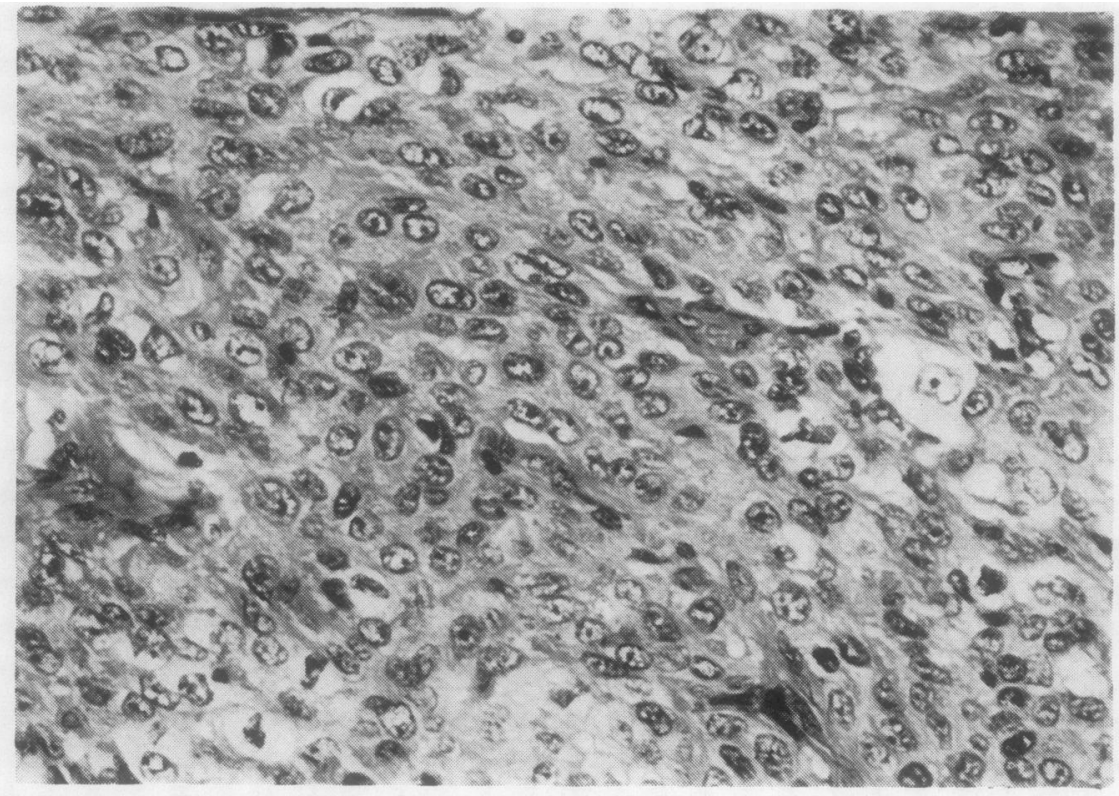

(a)

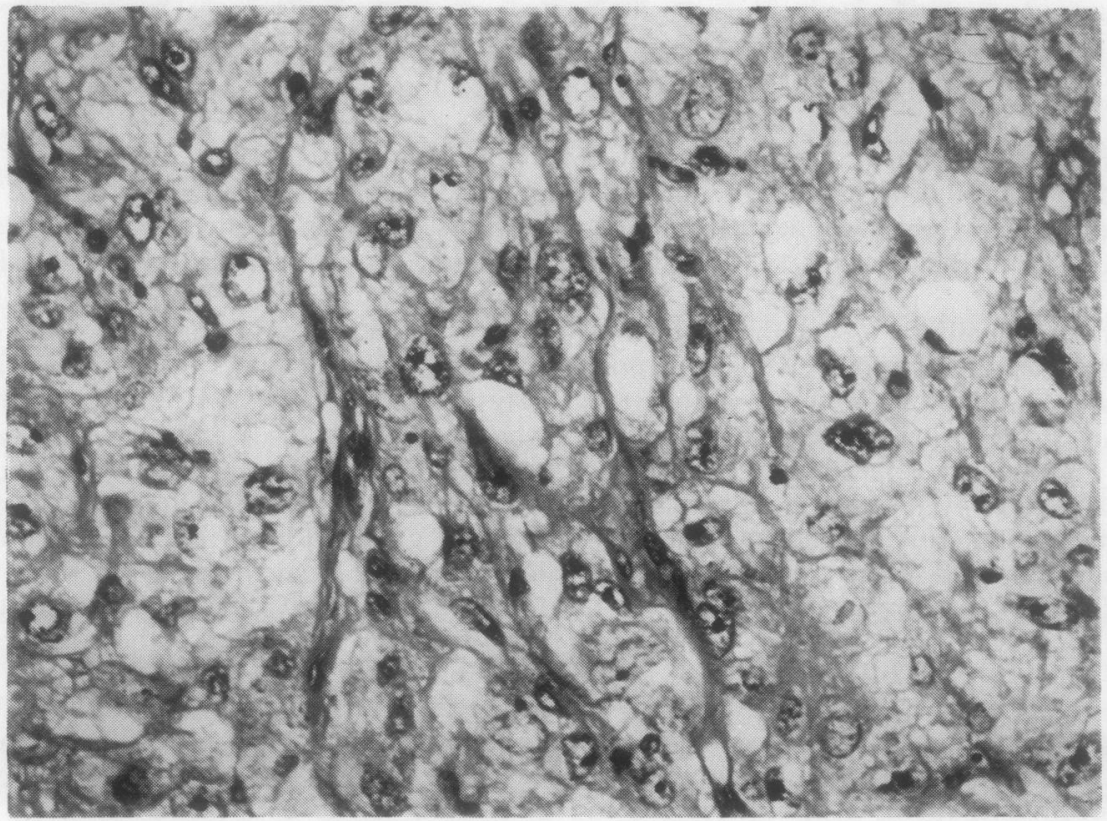

(b)

Fig. 12 Case 7. Two different tumours in the kidney. This patient died with metastases from adenocarcinoma: (a) tumour composed of cells with acidophilic cytoplasm; (b) tumour composed of cells with foamy cytoplasm. Both $H$ and $E \times 410$. 
lecting ducts which have become obstructed as a result of the surrounding interstitial fibrosis. Alternatively, it is possible that collecting tubules may be obstructed by oxalate crystals which are plentiful in this condition. It is unlikely that cyst formation is directly related to severe vascular changes as these are just as frequent in granular contracted kidneys as in cystic kidneys in chronic renal failure. There is no evidence that cystic transformation is associated with any specific type of nephritis although it must be added that the nature of the original disease was not known in all these patients, no less than five presenting with chronic uraemia and the diagnosis of chronic glomerulonephritis. In none of the patients in whom a renal biopsy was performed was there any indication of cyst formation. It must be assumed that cysts are a late manifestation of the disease. Enlargement of the kidneys was by no means the rule in these patients and, although it was a prominent feature in three cases (see Table), they may be normal or reduced in size. Curiously enough, in congenital adult polycystic disease treatment by long-term intermittent haemodialysis appears to cause a decrease in renal size (Thaysen et al., 1975).

Two important complications, haemorrhage and tumour formation, may occur as a result of cystic transformation of the kidneys in uraemic patients. Haemorrhage into the cysts is a frequent finding but it is only when it gives rise to painful enlargement of the kidney or haematuria or bursts into the retroperitoneal tissues that it becomes clinically apparent. In this connection the observations of Cabaluna and Eisinger (1974) are of interest. They described eight patients with advanced renal failure who developed painless gross haematuria after between one and 36 months on intermittent dialysis, despite the fact that haematuria had not been a feature of their original renal disease. Details of the pathology of the kidneys in their cases are not given but it is possible that the haemorrhage could be the result of cyst or tumour formation. Further factors likely to give rise to haemorrhage are clotting defects secondary to uraemia and, more likely, the use of heparin or longterm anticoagulants which are widely employed in dialysis patients.

It is recognised that patients who have undergone renal transplantation carry an increased risk of developing malignant tumours. Barnes et al. (1975) record the experiences of the Human Renal Transplant Registry and list 192 malignant neoplasms but do not include any primary renal tumours. What is not so generally known is that patients with uraemia who have not received a renal transplant also carry an increased risk of malignancy. In an interesting study of 646 uraemic patients Matas et al. (1975) note that there is a significant increase in cancer in uraemic patients and record two instances of primary renal carcinoma occurring before renal transplantation. The occurrence of multiple renal tumours has not received attention. In our experience, these are a complication of cystic change; they were present in five out of 14 cases recorded here and were not found in any case without numerous cysts. One reason that they may have been ignored relates to the difficulty in deciding whether such lesions are malignant or not. As the histological appearances give little indication of the way such tumours behave it is advisable to regard all lesions as potentially malignant, even when they have a uniform cytology and conform to the pattern of a clear cell or papillary 'adenoma'.

\section{References}

Barnes, B. A., Bergan, J., J., Braun, W. E., Fraumeni, J. F., Jr., Kountz, S. L., Mickey, M. R., Rubin, A. L., Simmons, R. L., Stevens, L. E., and Wilson, R. E. (1975). The 12th report of the Human Renal Transplant Registry. Journal of the American Medical Association, 233, 787-796.

Cabaluna, C., and Eisinger, R. P. (1974). Gross haematuria as a manifestation of advanced glomerular disease. Nephron, 12, 59-62.

Heptinstall, R. H. (1968). Pathology of end-stage kidney disease. American Journal of Medicine, 44, 656-663.

Heptinstall, R. H. (1974). Pathology of the Kidney, 2nd edition, p. 484 et seq. Little, Brown, Boston.

Matas, A. J., Simmons, R. L., Kjellstrand, C. M., Buselmeier, T. J., and Najarian, J. S. (1975). Increased incidence of malignancy during chronic renal failure. Lancet, 1, 883-886.

Thaysen, J. H., Christensen, E., Alarcon-Zurita, A., and Movild, B. (1975). Involution of polycystic kidneys during active treatment of terminal uremia. Acta Medica Scandinavica, 197, 257-260. 